\title{
A study for a MDO Process Applied to Conceptual Design of a Remotely Piloted Aircraft
}

\author{
Maria Luiza Cassão Gatelli ${ }^{1}$, Carlos Eduardo de Souza ${ }^{2}$, and Marcos Daniel de Freitas Awruch ${ }^{3}$ \\ ${ }^{1}$ Aerospace Engineering Course \\ ${ }^{2,3}$ Mechanical Engineering Department \\ 1,2,3 Federal University of Santa Maria (UFSM), Santa Maria, RS - Brazil. \\ E-mail: marialuizagatelli@gmail, carlos.souza@ufsm.br, marcos.awruch@ufsm.br
}

\begin{abstract}
The aircraft design process involves the interaction among disciplines with different nature. Finding an optimal concept is a trade off task that requires time and experience from designers and engineers. Multidisciplinary Design optimization (MDO) methods help to automatise and to simplify that complex task, aiming to obtain design solutions that comply with multiple requirements and constraints at once. The present work seeks to develop a conceptual design framework of a remotely piloted aircraft (RPA). A review on this subject is presented, identifying different MDO approaches. The analysed disciplines of aeronautical design are: geometry, aerodynamics, weight, performance, stability and flight dynamics. The relationship among them are presented in a block diagram. Afterwards, a technique of MDO is applied to the framework, assembling a mono objective problem using the Particle Swarm optimization (PSO) algorithm to minimise aircraft structural mass under stability constraints. This objective is obtained from dimensional parameters of feasible aircraft concepts as output solution of the process. Therefore, the MDO method speeds the design process up, without employing the traditional trial and error approach, which, as well as doesn't guarantee the achievement of an optimal configuration, turns the process slow and expensive.
\end{abstract}

Keywords: Conceptual design. MDO. optimization. PSO. RPA.

\section{Introduction}

The global market and international competition lead aeronautical industry to continuously improve its performance in the engineering design process [1]. As described by Raymer [2], the aircraft design process can be divided into three major phases: conceptual, preliminary and detail design. The design changes have a lower cost in the conceptual phase than in the subsequent ones, following a trend shown in Fig. 11 This figure presents the cost increasing as the project advances in time and also how the impact of design changes decreases as the level of detail increases.

Aiming to reduce time and improve design confidence level, the application of new design automated methods is welcome. "As the aircraft design is multidisciplinary by nature, the need to apply Multidisciplinary Design optimization (MDO) methods is well understood and accepted" [4]. "The main motivation for using MDO is that the performance of a multidisciplinary system is driven not only by the performance of the individual disciplines but also by their interactions" [5].

Furthermore, the operation of remotely piloted aircraft (RPA) is drawing an increasing attention in military and civil sectors because of the absence of human operators on board,

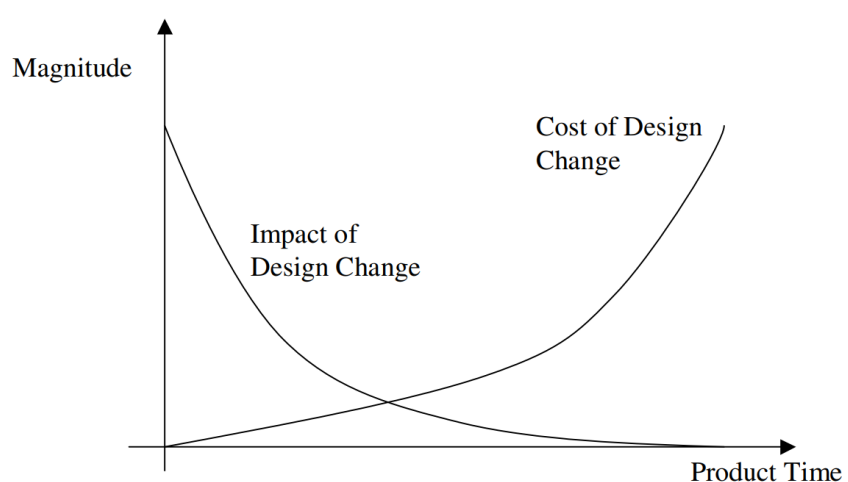

Figure 1: Cost and impact of design change over product time, adapted from [3].

what reduces operating costs and expands capabilities. These aircraft are also called unmanned aerial vehicle (UAV) and defined according to the US Department of Defence (DoD) as: "a powered, aerial vehicle that does not carry a human operator, uses aerodynamic forces to provide vehicle lift, can fly autonomously or be piloted remotely, can be expendable or recoverable, and can carry a lethal or nonlethal payload. Bal- 
listic or semi ballistic vehicles, cruise missiles, and artillery projectiles are not considered unmanned aerial vehicles" [6].

The application of RPA, which had been largely military since the 1950s, has expanded into the civilian market. Tasks such as battlefield observation and surveillance have been replaced by monitoring of urban areas, plantations and power transmission lines or simply recreation, in the form of model aircraft with imaging systems. Table 1 lists examples of civilian uses of RPA.

Table 1: Examples of civilian uses of RPA, adapted from [7].

\begin{tabular}{|c|c|}
\hline Area & Example \\
\hline Aerial Photography & Films and advertising videos \\
\hline Agricult & $\begin{array}{l}\text { Crop monitoring and spraying and } \\
\text { herd monitoring and driving }\end{array}$ \\
\hline Coastguard & $\begin{array}{l}\text { Search and rescue, coastline and } \\
\text { sea-lane monitoring }\end{array}$ \\
\hline Conservation & Pollution and land monitoring \\
\hline Energy Industry & $\begin{array}{l}\text { Power line inspection, wind turbine } \\
\text { inspection }\end{array}$ \\
\hline $\begin{array}{l}\text { Fire Services and } \\
\text { Forestry }\end{array}$ & Fire detection and incident control \\
\hline Gas and Oil Ind. & Land survey an \\
\hline Information Services & $\begin{array}{l}\text { News information and pictures, fea- } \\
\text { ture pictures, e.g. wildlife }\end{array}$ \\
\hline Lifeboat Ins & $\begin{array}{l}\text { Incident investigation, guidance } \\
\text { and control }\end{array}$ \\
\hline $\begin{array}{l}\text { Local and Police Au- } \\
\text { thorities }\end{array}$ & $\begin{array}{l}\text { Survey, disaster control, search for } \\
\text { missing persons, security and incid- } \\
\text { ent surveillance }\end{array}$ \\
\hline Meteorology & $\begin{array}{l}\text { Sampling and analysis of atmo- } \\
\text { sphere for forecasting }\end{array}$ \\
\hline Traffic Agencies & $\begin{array}{l}\text { Monitoring and control of road } \\
\text { traffic }\end{array}$ \\
\hline Ordn & Aerial photography for mapping \\
\hline Survey Organisations & $\begin{array}{l}\text { Geographical, geological and ar- } \\
\text { chaeological survey }\end{array}$ \\
\hline $\begin{array}{l}\text { Water Boards } \\
\text { etc. }\end{array}$ & Reservoir and pipeline monitoring \\
\hline
\end{tabular}

Another application of RPA is as research platform in academic and industrial spaces, aiming to develop aeronautical knowledge and employee qualification. Two factors are of key importance: the reduced dimensions allow the aircraft to be produced in small laboratories and by few people and the costs are very small when compared to the development of a manned aircraft. All electronic components, for example, are readily available from online stores, and a small specialization is needed to integrate a complete airframe.

The present work focuses on establishing a MDO process dedicated to the design of RPA and displays the recent advances in that purpose. The next section presents a brief literature review, followed by the proposed methodology. The numerical studies section details some simulations and optimization results applied to a small aircraft design.

\section{Bibliography review}

"A remotely piloted aircraft system (RPAS) comprises a number of subsystems which include the aircraft, its payloads, the control station(s) (and, often, other remote stations), aircraft launch and recovery subsystems where applicable, support subsystems, communication subsystems, transport subsystems, etc." [7].

Sobieszczanski-Sobieski and Haftka [8] presented a revision about multidisciplinary optimization in aerospace design, focusing on the multiple ways that engineers deal with the main challenges of a MDO process: computational cost and organisational complexity. According to the authors, it's possible to identify three categories of MDO problems. The first includes problems with two or three interactive disciplines, where a single analyst might acquire all necessary knowledge. At analysis level, this can lead to the creation of a new discipline responsible for the interaction between the involved disciplines, as aeroelasticity or thermoelasticity. The next category includes works in which the multidisciplinary optimization of the entire system is executed at a conceptual level by simple analysis tools. The third category includes problems that focus on organisation and computational challenges and develops techniques able of solving them.

Sobester and Keane [9] proposed a Computer Aided Design (CAD),based UAV conceptual design framework and discussed some of the challenges arising at the conceptual level. The design workflow was described from the mission profile until the structural model and flow analysis. The basis of MDO study was the flight envelope resulted from the performance analysis of the aircraft. The results of six MDO iterations were achieved combining three different values of negative twist and two values of outboard sweep angle, showing the maximum wingtip deflections versus the maximum $L / D$ ratios. The authors concluded that commercial, off-theshelf, CAD tools could be integrated into the design process as early as the conceptual level, providing the models required by the various strands of multidisciplinary analysis.

Landolfo [10] outlined a structural and aerodynamic design of an UAV with multiple lift surfaces. The author investigated if a particular nonplanar wing concept could achieve the requirements of the mission of a small reconnaissance, surveillance and target acquisition UAV. The results, compared under varying assumptions specific to an equivalent monoplane and biplane, suggest that potential efficiency gains for the new configuration may be possible using the nonplanar wing configuration under explicit conditions. However, a conventional monoplane concept is suggested, given the areas of study covered in the paper.

Lundstrom et al. [11] proposed a distributed framework for micro aerial vehicle (MAV) design automation. A discrete propulsion system modelling is combined with a parametric CAD model and a panel code for aerodynamic performance prediction. The design optimization has been divided into two successive parts through a genetic algorithm. The first one runs without invoking $\mathrm{CAD}$ and aerodynamic analysis tools until convergence is achieved. The second part uses the pre- 
vious optimum solution as the starting point to consider these time-costly software. The process runs until the results from the first and second part agree. The author suggests that the design framework is a helpful tool in MAV design. However, it is necessary to ensure the proper balance and size restrictions are met and the MAV must have large enough volume to accommodate its intended components.

Agte et al. [12] affirmed that the origins of MDO are found in the development of structural optimization. The inclusion of other disciplines into the process was a natural evolution, since the aerodynamics, propulsion and performance variables, for example, are intrinsically related to the structural efficiency. Initial processes were developed in sequential levels, where variables and objective functions were uncoupled, and later it was possible to study in a single optimization block with as much as possible coupling among disciplines.

Medeiros [13] developed an UAV for monitoring and sensing agricultural activities. The methodology applied to the prototype construction, the UAV development, the monitoring and processing of captured aerial images are presented in this master's thesis. The results are considered satisfactory due to the design requirements and to the image quality of tillage areas.

Martins and Lambe [5] performed extensive research about MDO architectures, classifying them in monolithic (a single optimization problem is solved) and distributed (the same problem is partitioned into multiple subproblems). A unified description with terminology, mathematical notation and diagram development was provided aiming to compare the benefits and drawbacks of each category. The authors suggest that it is necessary to test multiple architectures on a given MDO problem to determine which one is most efficient for each case. The work is a powerful tool to find the most appropriate architecture for the optimization problem.

Elmendorp et al. [14] developed a conceptual design method and analysis of conventional and unconventional aircraft. Initially, the top-level requirements (TLRs) in combination with a chosen design configuration and design objective are translated into a first estimate of the aircraft's geometry and its associated key performance indicators. A fully parametrized multi-model generator generates input data for the disciplinary analysis tools, including weight, aerodynamics, landing gear sizing, engine and cost. The verification of the software is performed by comparing the output data of a conventional configuration aircraft with another reference aircraft with similar TLRs, resulting in satisfactory error rates. The authors also analysed four different configurations demonstrating the variety of the configurations that may be designed and analysed as well as the validity of the comparison.

Bryson et al. [15] presented a multidisciplinary design analysis and optimization of quiet small unmanned aerial systems (SUASs). A multi-objective, non dominated sorting genetic algorithm (NSGA-II) was used to find the Paretooptimal trade space for maximising range and minimising vehicle noise. The design variables were wing area, aspect ratio, taper ratio, maximum camber, maximum camber location, maximum thickness and loiter speed. The constraints were angle of attack, propulsion weight, fuel weight and thrust required. The study provided suitable fidelity for early (conceptual to preliminary) design and ratified noise as a discipline integrated at the conceptual design level.

Hosseini et al. [16] applied a decoupling Uncertainty-based Multidisciplinary Design optimization (UMDO) method to design UAVs as a case study, using a genetic algorithm optimization method and sequential quadratic programming (SQP). The optimizer is located at system level within which UAV design parameters (a total of 37) are achieved in a way that, observing the problem's constraints (a total of 33), optimization criterion (overall mass of the UAV) becomes minimum. In order to provide an algorithm without uncertainties, it was necessary to identify the doubtful sources of the parameters, eg fuel consumption and aerodynamic coefficients. Then, the method proposed by the authors was applied to a study case, generating an increase in the objective function of the problem (mass). This result was justified as an algorithm way out to compensate for failure probability and elevate the chance of success to $100 \%$.

Papageorgiou et al. [17] developed a MDO framework applied to UAV design focusing on radar signature and sensor performance considering also flight trajectory. The optimization problem of the case-study considered was about improving the performance of the aircraft over a specified mission while simultaneously considering the efficiency of the stealth and surveillance systems. One of the objectives was the minimisation of the Maximum Take-Off Weight (MTOW). The proposed MDO framework took into account aircraft's geometry, aerodynamics, trim, stability and simulation of the mission. The solution of the present optimization problem was achieved by implementing a single and a multi-level decomposition architecture. The proposed multidisciplinary optimization and analysis framework had the potential to significantly enhance the performance of the design when mission, stealth, and surveillance requirements must be considered.

Analysing the references cited previously, a work like Landolfo [10] elucidates the difficulties found during the unmanned aircraft design process, due to the lack of availability and reliability of historic regressions and design coefficients. "The potential consequences of early design decisions made with insufficient data include missed opportunities to increase vehicle performance and cost overruns to meet performance metrics or correct design defects" [15].

It is explicit the dependence between the mission requirements and the methodology adopted during the design process and the chosen optimization method. According to Martins and Lambe [5], there are many architectures available to solve the optimization problems, and they must be presented in diagrams, such as those found in Bryson et al. [15], Hosseini et al. [16] and Papageorgiou et al. [17]. It is notorious that the disciplines related to airworthiness, like propulsion, mass, structures, aerodynamics and stability, receive more attention in an optimization framework than disciplines that are not in the field of aeronautics. Hosseini et al. [16] and Papageorgiou et al. [17] integrated into their framework 
non-conventional disciplines like noise and radar signature, respectively, demonstrating the potential of an embracing architecture integrated in an optimization process during the aircraft conceptual design phase.

\subsection{Design phases}

The division of the aircraft design into the three major phases, already cited above, is important to establish limits for decision making regarding to passing to subsequent phases and finally to manufacturing. In the first phase, the conceptual design, the objective is to develop alternative concepts that meet the problem solution. No excessive details should be taken into account in this phase since it would delay the process but also add many constraints. The second phase, the preliminary design, performs the definition of product layout configuration, technical and economically analysed. The detailed design, the third phase, is responsible for its final detailing, including meticulous documentation of the product and planning of the manufacturing process.

\subsection{Design requirements}

The customer's needs have to be translated into design requirements, mostly through customer plus engineering requirements. The first ones refer to objectives as articulated by the customer or client. The engineering ones refer to the design and performance parameters that can contribute to achieving the customer requirements [18]. The system engineering is fundamental in this process, because, as design requirements are a formalisation of all basic characteristics that involve an aircraft design, gross mistakes might occur if those are not well defined.

The aircraft mission defines the payload onboard in a determined velocity and range, utilising a certain type of engine. "The functional analysis is a process of translating system requirements into detailed design criteria and the subsequent identification of the resources required for system operation and support" [18]. Examples of functional criteria of RPA design includes maximum weight or span, operating altitude, range, fuselage volume, launching method, etc.

\subsection{Multidisciplinary design optimization}

The MDO technique was developed for complex engineering systems involving multiple disciplines or subsystems. The space design is explored in a form that the variables of interest are optimized under some constraints, simultaneously and automatically, until a satisfactory design point for all disciplines is found.

According to Agte et al. [12], the general formulation of an optimization problem can be written as

$$
\begin{array}{ll}
\min & f(\mathbf{x}, \mathbf{p}) \\
& \mathbf{x}=\left[x_{1}, \ldots x_{n}\right]^{T}, \quad \mathbf{p}=\left[p_{1}, \ldots p_{m}\right]^{T} \\
& x_{i, L . B .} \leq x_{i} \leq x_{i, U . B .}, i=1,2, \ldots n \\
\text { s.t. } & \mathbf{g}(\mathbf{x}, \mathbf{p})<0, \quad \mathbf{h}(\mathbf{x}, \mathbf{p})=0,
\end{array}
$$

where $f$ is the objective function, $\mathbf{x}$ is a $n$-dimensional vector of design variables with lower and upper bounds, $\mathbf{p}$ is a vector of fixed parameters that influence the behavior of the system but cannot be freely chosen (material properties, operating conditions,..), and $\mathbf{g}$ and $\mathbf{h}$ are vectors of inequality and equality constraints, respectively. However, when dealing with multiple disciplines, it might be a difficult task to solve the minimization problem at once, and different strategies must be sought.

One of the most important considerations when implementing MDO is to find a way of organising each discipline analysis models, simplified models and the optimization method agreeing with a correct problem formulation, as discussed by Martins and Lambe [5]. The MDO architecture is the combination of the problem formulation with the organisational strategy, allowing to identify how the different models are coupled and how the overall optimization problem is solved, depending if its structure is monolithic or distributed. This organisation itself is a challenge. The next section describes the strategy and framework proposed in the present work.

\section{Proposed methodology}

This works aims to develop a conceptual design tool of RPA integrated with an optimization algorithm. At this stage of the work, it is applied to a Mini UAV category, which is capable of being hand-launched and operating at ranges up to $30 \mathrm{~km}$, according to Austin [7]. The tool considers aircraft with conventional configuration tractor or pusher with a payload bay with a single tail boom leading to a conventional, cruciform or " $\mathrm{T}$ " tail configuration without landing gear and electric propulsion system.

The first step to develop the design tool is to outline clearly the objectives of the conceptual design phase. Then, it's necessary to organise a framework aiming to reach a viable concept that meets the mission requirements. The framework structure depends upon the conceptual design process of unmanned aircraft. It can be expressed through a block diagram relating the various disciplines addressed in aircraft design such as aerodynamics, weight and stability. Afterwards, the optimization method can be applied, defining the project variables, objective function and constraints.

\subsection{Remotely piloted aircraft conceptual design}

The unmanned aircraft design process differ from the manned ones, once the information source, historic regressions and design coefficients are not available or are not reliable [19]. In this work, the following disciplines of aeronautical design are analysed: geometry, aerodynamics, weight, performance, stability and flight dynamics. The wing and tail geometry are calculated according to Gudmundsson [20]. Weight prediction is based on the volume calculation of each structural component and multiplied by a material density. Aerodynamics coefficients are calculated using Roskam Class II methodology [21]. Performance estimates are found from Traub [22] formulation. Stability derivatives are predicted from empirical data presented in Roskam [23]. Flight dynamics are analysed based on Cook [24] methodology. 


\subsection{Particle swarm optimization}

"A PSO algorithm explores the design space of the objective function by adjusting the trajectories of the individual agents, also called particles, as these trajectories form piece wise paths in a quasi-stochastic manner" [25]. The movement of a swarm particle consists of two components: one stochastic and another deterministic. Each particle is attracted toward the position of the current global best $\mathbf{b}^{*}$ and its own best location $\mathbf{x}_{\mathbf{i}}^{*}$ in history, while at the same time it has a tendency to move randomly. The aim is to find the global best among all the current best solutions until the objective no longer improves or after a certain number of iterations.

The position vector $\mathbf{x}_{\mathbf{i}}$ and the velocity $v_{\mathbf{i}}$ for the particle $i$ are utilised to determine the new velocity vector as

$$
v_{\mathbf{i}}^{t+1}=v_{\mathbf{i}}^{t}+\alpha \varepsilon_{\mathbf{1}} \odot\left[\mathbf{b}^{*}-\mathbf{x}_{\mathbf{i}}^{t}\right]+\beta \varepsilon_{\mathbf{2}} \odot\left[\mathbf{x}_{\mathbf{i}}^{*}-\mathbf{x}_{\mathbf{i}}^{t}\right]
$$

where $\varepsilon_{1}$ and $\varepsilon_{2}$ are two random vectors that vary between 0 and 1 and the parameters $\alpha$ e $\beta$ are acceleration constants.

The initial velocity for the particle may be taken as zero $v_{\mathbf{i}}^{t=0}=0$. Then, the new position is updated as

$$
\mathbf{x}_{\mathbf{i}}^{t+1}=\mathbf{x}_{\mathbf{i}}^{t}+v_{\mathbf{i}}^{t+1} .
$$

According to Yang [25], the pseudo code of a PSO algorithm can be written as follows:

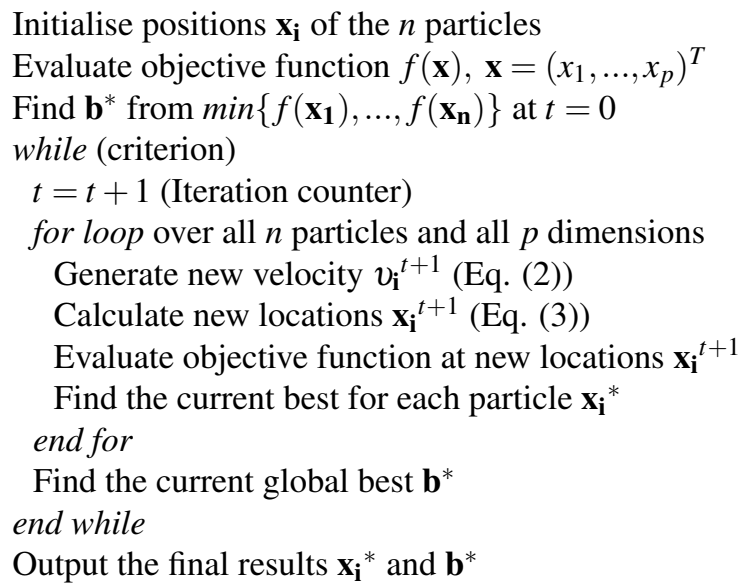

In order to add constraints to the PSO problem, it's possible to transform it into an unconstrained problem by using the penalty method. When some constraint is not respected, a penalty parameter may be used, for example,

$$
\lambda=e^{10|\mathbf{g}(\mathbf{x})|} .
$$

Therefore, a corresponding value $\lambda$ for each constraint will penalize the computed objective, turning the penalty function into the new objective function $\lambda f(\mathbf{x})$.

\subsection{Definition of the optimization problem}

This paper aims to investigate a mono objective constrained optimization utilising a PSO technique. The optimization problem must be assembled according to Eq. 1, resulting in

$$
\begin{array}{ll}
\min & f(\mathbf{x}, \mathbf{p})=M_{\text {tot }} \\
& x_{i, L . B .} \leq x_{i} \leq x_{i, U . B .}, i=1,2, \ldots 9 \\
\text { s.t. } & \\
& C_{m_{\alpha}}<0, \\
& C_{n_{\beta}}>0, \\
& 12.5 \%<S M<17.5 \% \\
& \lambda_{A l p}<0 \quad e \quad \lambda_{A l g}<0 .
\end{array}
$$

In the above problem, $\mathbf{x}$ and $\mathbf{p}$ are the design variables and parameters vector, defined as

$$
\mathbf{x}=\left[\begin{array}{c}
A R \\
S \\
\lambda \\
\Lambda_{L E} \\
x_{r_{w}} / l_{f u s} \\
\lambda_{f u s} \\
A R_{H T} \\
A R_{V T} \\
l_{H T} / \bar{c}
\end{array}\right] \quad \text { and } \quad \mathbf{p}=\left[\begin{array}{c}
H \\
V_{\infty} \\
V_{H T} \\
V_{V T} \\
V_{\text {ool }} \\
C_{\text {Bat }} \\
\rho_{M a t} \\
M_{P L} \\
\vdots
\end{array}\right]
$$

The components of the vector of design variables $\mathbf{x}$ are described in Table 2 The vector $\mathbf{p}$ of design parameters considers characteristics relating to tail and fuselage geometry, aerodynamics, propulsion, flight control and materials. Some of the parameters are listed in Table 3

The constraints considered in this study are presented in Table 4 and correspond to flight quality parameters. These are just a small set of constraints, but play an important role in the final design configuration. This is a key condition for future improvements, since constraints such as aeroelastic conditions (divergence and flutter) or structural response (wing root stress) can be easily implemented.

Table 2: Design variables of the optimization problem in study.

\begin{tabular}{lll}
\hline Area/No & Design variable & Symbol \\
\hline \multicolumn{3}{l}{ Wing geometry } \\
1 & Aspect ratio & $A R$ \\
2 & Area & $S$ \\
3 & Taper ratio & $\lambda$ \\
4 & Sweep of the leading edge & $\Lambda_{L E}$ \\
5 & Wing root location relative to fuselage & $x_{r_{w}} / l_{f u s}$ \\
& length & \\
& Fuselage & $\lambda_{f u s}$ \\
6 & Fineness ratio & \\
& Tail geometry & $A R_{H T}$ \\
7 & Aspect ratio of horizontal tail & $A R_{V T}$ \\
8 & Aspect ratio of vertical tail & $l_{H T} / \bar{c}$ \\
9 & Distance of both horizontal tail $\bar{c} / 4$ to & \\
& wing $\bar{c} / 4$ along the x-axis relative to $\bar{c}$ & \\
\hline
\end{tabular}

These disciplines are organised in a $N \times N$ design diagram in order to outline the inputs and outputs regarding to each dis- 
Table 3: Design parameters of the optimization problem in study.

\begin{tabular}{lll}
\hline Area/№ & Design parameter & Symbol \\
\hline & Tail geometry & \\
1 & Tail boom radius & $r_{T B}$ \\
3 & Horizontal tail volume coefficient & $V_{H T}$ \\
4 & Vertical tail volume coefficient & $V_{V T}$ \\
& Fuselage & \\
10 & Internal volume & Vol $l_{f u s}$ \\
& Aerodynamics & \\
11 & Operating altitude & $H$ \\
12 & Cruise velocity & $V_{\infty}$ \\
17 & Wing airfoil & - \\
18 & Tail airfoil & - \\
& Propulsion & \\
19 & Electric motor mass & $M_{M o t}$ \\
20 & Electric motor power & $P_{M o t}$ \\
& Flight control & \\
23 & Battery capacity & $C_{B a t}$ \\
25 & Battery mass & $M_{B a t}$ \\
26 & Servomotor mass & $M_{S r v}$ \\
31 & Materials & $\rho_{M a t}$ \\
34 & Density & $M_{P L}$ \\
\hline & Payload &
\end{tabular}

Table 4: Design constraints of the optimization problem in study.

\begin{tabular}{lll}
\hline Area/No $\quad$ Constraint & Equation \\
\hline 1 & $\begin{array}{l}\text { Stability } \\
\text { Pitching moment due to } \\
\text { angle of attack derivative }\end{array}$ & $C_{m_{\alpha}}<0$ \\
2 & $\begin{array}{l}\text { Yawing moment due to } \\
\text { sideslip derivative }\end{array}$ & $C_{n_{\beta}}>0$ \\
3 & $\begin{array}{l}\text { Limits of static margin } \\
\text { Real part of eigenval- }\end{array}$ & $12.5 \%<S M<17.5 \%$ \\
4 & $\lambda_{\text {long }}<0$ and $\lambda_{\text {lat }}<0$ \\
& $\begin{array}{l}\text { ues of matrix of longit- } \\
\text { udinal and lateral direc- }\end{array}$ & \\
&
\end{tabular}

cipline and also between them. This approach allows a multidisciplinary and integrated framework converging between subsystems.

The implemented code is organised according to the diagram shown in Fig. 2, that is an adaptation of a complete $N \times N$ diagram, with information running only in one direction. Here, the main design code calls each discipline from left to right, and thus the information to each block comes always in this direction. The final feedback comes from each block to the Design block, and then to the PSO algorithm. It means that there the result from each block on the right only comes back to a block on the left after updating data in the main design block.
The proposed framework is implemented completely in Python language and takes advantage of open source packages. All disciplines are implemented in specific functions, organised such that the input and output variables are available at the Design block. A 3D plotting function was also implemented to better visualise the final concept and identifies the general dimensions of the aircraft, as well as the centre of gravity position of the main parts.

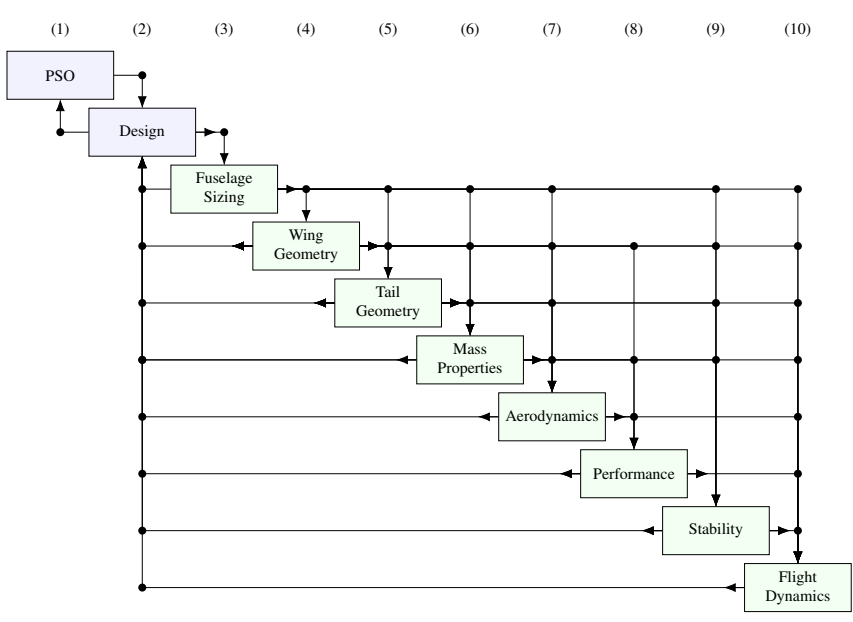

Figure 2: Proposed optimization diagram.

\section{Numerical studies}

The remotely piloted aircraft conceptual design tool is applied to the design of a small RPA. The aircraft described in 4.1 is considered as basis for the mission and design requirements for a case study. The application of the methodology is limited to conventional configurations, such as tube and wing, with electric propulsion.

\subsection{Reference model}

The chosen reference aircraft is the Spy Owl 200 Research Version presented in Figure 3 It is a commercial RPA designed exclusively for research. The main technical specifications of the Spy Owl 200 are presented in Table 5, from where stand out the Maximum Take-off Weight (MTOW) and span, including take-off and landing method, characteristics that have a great impact in the aircraft structural mass.

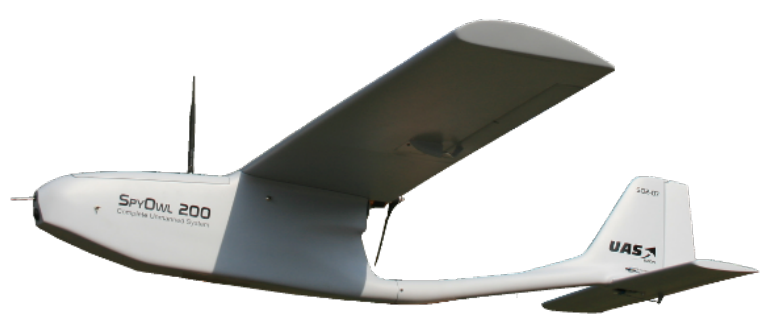

Figure 3: Spy Owl 200, a reference model for the present study, adapted from [26]. 
Table 5: Technical specifications of the reference aircraft, the Spy Owl 200, adapted from [26].

\begin{tabular}{ll}
\hline Characteristic & Value \\
\hline Wing span & $2.01 \mathrm{~m}$ \\
Length & $1.53 \mathrm{~m}$ \\
MTOW (Hand-launched) & $6.5 \mathrm{~kg}$ \\
Autonomy & 2 hours \\
Typical mission altitude & $75-1500 \mathrm{~m} \mathrm{AGL}$ \\
Maximum payload mass & $2.7 \mathrm{~kg}$ \\
including batteries & \\
Take-off method & Hand-launched \\
Landing method & Belly landing \\
Structure & Extremely robust full \\
& composite structure \\
Cruise speed & $14-22 \mathrm{~m} / \mathrm{s}$ \\
Maximum speed & $40 \mathrm{~m} / \mathrm{s}$ \\
\hline
\end{tabular}

\subsection{Design requirements}

The design requirements of the case study aircraft of a potential client proposed by the authors are presented below:

- The aircraft should be capable of being easily transported between operation sites;

- The aircraft should be light enough to be hand-launched;

- The aircraft should be recovered from a belly landing;

- The aircraft should be capable of being remotely piloted or to fly autonomously with interference of a human operator;

- The aircraft should transmit the flight data real-time for ground system.

The RPA mission is to carry a research payload with a determined mass utilising electric propulsion.

The functional requirements are:

- Maximum mass of $6.5 \mathrm{~kg}$;

- Maximum span of $2 \mathrm{~m}$;

- Cruise speed of $20 \mathrm{~m} / \mathrm{s}$;

- Hand-launched by the operator;

- Recovered by belly landing;

- Operating altitude of $100 \mathrm{~m}$.

RPA used as research platform in academic laboratories, for example, may have a reduced number of subsystems. The launching requirements induce the absence of landing gear, reducing system complexity. The transporting requirement between operating sites induces to the facility of carrying RPAS by an operator between one place and another. The maximum mass requirement is associated with the physical resistance of an operator responsible for the launching.

\subsection{Input data}

Initially, it is necessary to define the set of input variables, and their lower and upper bounds. Those values are presented in Table 2. The values of some of the design parameters vector $\mathbf{p}$ are listed in Table 7. Those values are fixed for the entire process.

At this point, there is still a need for a careful interference of the designer, since the limit values should be chosen based on engineering knowledge, and especially considering experience or known references. The limits of the wing and tail aspect ratio are estimated according to Gudmundsson [20]. The limits of wing area are defined by the authors based on the reference model. Gudmundsson [20] also presents reference values for taper ratio and sweep angle of the wing leading edge. The fuselage fineness ratio is estimated based on Nicolai and Carichner [27]. The variable $l_{H T}$ usually has a value between $3 \bar{c}$ and $6 \bar{c}$, where $\bar{c}$ is wing mean aerodynamic chord (MAC), Then, in tail geometry discipline, the variable $l_{H T}$ is multiplied by $\bar{c}$. The same procedure is adopted for $x_{r_{w}}$. The maximum value of this variable is $\left(l_{f u s}-c_{r}\right) / 2$, where $c_{r}$ is wing root chord.

Table 6: Lower and upper bounds of the design variables of the optimization problem in study.

\begin{tabular}{lllll}
\hline № & Variable & $x_{i, L . B .}$ & $x_{i, U . B .}$ & Unit \\
\hline 1 & $A R$ & 6 & 11 & {$[-]$} \\
2 & $S$ & 0.05 & 0.5 & {$\left[\mathrm{~m}^{2}\right]$} \\
3 & $\lambda$ & 0.3 & 1 & {$[-]$} \\
4 & $\Lambda_{L E}$ & 0 & 10 & {$[\mathrm{deg}]$} \\
5 & $x_{r_{w}} / l_{f u s}$ & 0 & 1 & {$[-]$} \\
6 & $\lambda_{f u s}$ & 3 & 6 & {$[-]$} \\
7 & $A R_{H T}$ & 3 & 5 & {$[-]$} \\
8 & $A R_{V T}$ & 1 & 2.5 & {$[-]$} \\
9 & $l_{H T} / \bar{c}$ & 3 & 6 & {$[-]$} \\
\hline
\end{tabular}

The values of $V_{H T}$ and $V_{V T}$ are the same utilised by Landolfo [10]. The tail taper ratio and sweep of the leading edge are chosen in order to simplify the design. The fuselage volume is based on the volume of propulsion and flight system components and in an estimate of payload mass.

In order to estimate the aircraft centre of gravity, a table with the estimated location in $x, y$ e $z$ and mass of each of the basic component is assembled and is updated through the process. This allows updating the aircraft mass and inertia properties for each design.

\subsection{Minimisation of aircraft structural mass}

Since propulsion and flight control systems are fixed, as well as payload, the objective is to minimise aircraft structural mass, finding the optimal geometry that respect all constraints. To start the optimization, the number of particles is 50. There are two stop criteria. The first one is related to a minimum number of iterations that the best value of the objective function is not updated. It means that if the best value of the global objective function remains the same for 
Table 7: Fixed design parameters of the optimization problem in study.

\begin{tabular}{|c|c|c|c|}
\hline Area/№ & Parameter & Value & Unit \\
\hline & Tail geometry & & \\
\hline 1 & $r_{T B}$ & 0.015 & [m] \\
\hline 3 & $V_{H T}$ & 0.675 & {$[-]$} \\
\hline 4 & $V_{V T}$ & 0.0375 & {$[-]$} \\
\hline 10 & $\begin{array}{l}\text { Fuselage } \\
\text { Vol }{ }_{f u s} \\
\text { Aerodynamics }\end{array}$ & 0.0062 & {$\left[\mathrm{~m}^{3}\right]$} \\
\hline 11 & $H$ & 100 & [m] \\
\hline 12 & $V_{\infty}$ & 20 & {$[\mathrm{~m} / \mathrm{s}]$} \\
\hline 17 & Wing airfoil & NACA2412 & {$[-]$} \\
\hline 18 & $\begin{array}{l}\text { Tail airfoil } \\
\text { Propulsion }\end{array}$ & NACA0012 & {$[-]$} \\
\hline 19 & $M_{M o t}$ & 0.082 & {$[\mathrm{~kg}]$} \\
\hline 20 & $\begin{array}{l}P_{M o t} \\
\text { Flight Control }\end{array}$ & 438 & [W] \\
\hline 23 & $C_{\text {Bat }}$ & 3 & {$[\mathrm{Ah}]$} \\
\hline 25 & $M_{B a t}$ & 0.346 & {$[\mathrm{~kg}]$} \\
\hline 26 & $\begin{array}{l}M_{S r v} \\
\text { Materials }\end{array}$ & 0.1 & {$[\mathrm{~kg}]$} \\
\hline 31 & $\begin{array}{l}\rho_{M a t} \\
\text { Payload }\end{array}$ & 1420 & {$\left[\mathrm{~kg} / \mathrm{m}^{3}\right]$} \\
\hline 34 & $M_{P L}$ & 0.5 & {$[\mathrm{~kg}]$} \\
\hline
\end{tabular}

1000 iterations, the code considers it as a convergence. The second stop criteria is related to the standard deviation of the last 1000 best global values of the objective function. If this standard deviation value is lower than 0.001 , the code also converges.

Objective function iteration histories for 10 runs are shown in Fig. 4. The different initial values are due to random estimates of the PSO. The evaluations of the objective function (artificial value of the aircraft mass due to penalty factors) varied within 1.64 and $1.8 \mathrm{~kg}$, resulting in an error of approximately $9.8 \%$ for this analysis.

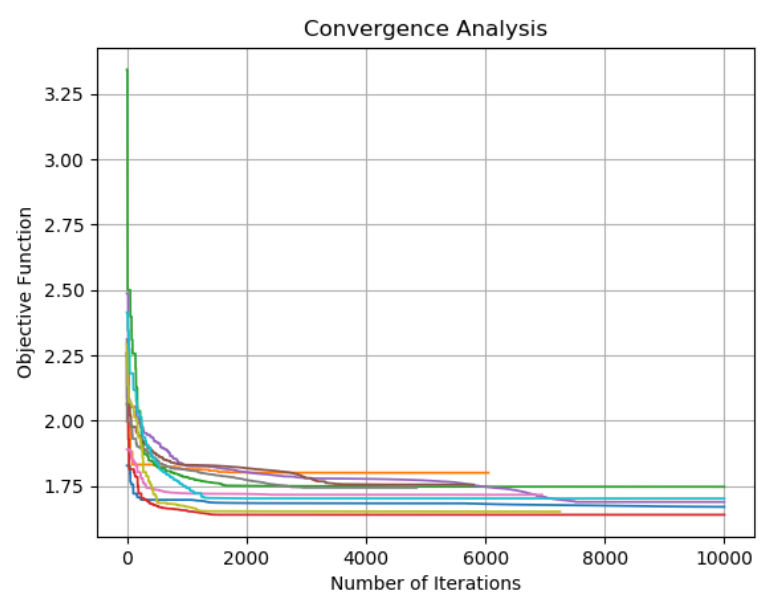

Figure 4: Objective function iteration history.

\subsection{Final concept}

The final result obtained from the optimization defines an aircraft concept, which should be the basis for the next design phases. The history of iterations of the global objective function for the optimized concept is presented in Fig. 5 More than 4000 iterations were necessary to achieve the optimal result. That means one hour running in a personal notebook, with an Intel Core i5-4210U processor, at $1.7 \mathrm{GHz}$.

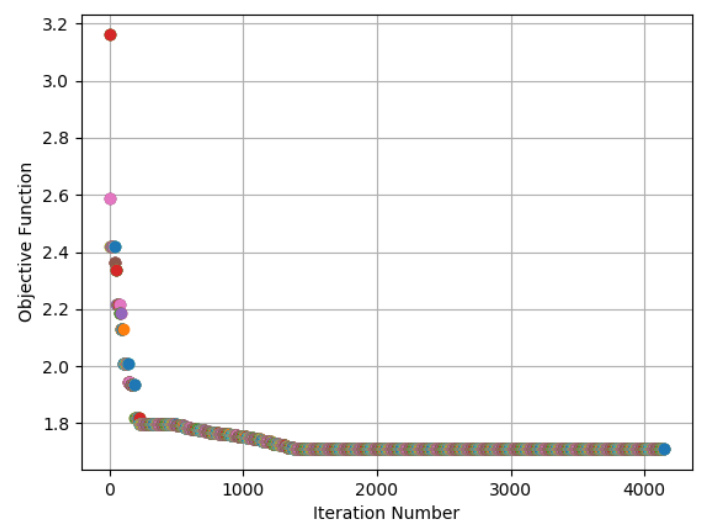

Figure 5: Objective function iteration history of the final concept.

Figures 6 to 8 show the evolution of aircraft configuration at different iteration steps of history shown in Fig. 5. The aircraft configuration at iteration number 1 is presented in Fig. 6 The length and span dimensions are larger than the following cases, what impacts in the total weight. This configuration doesn't respect all imposed constraints, especially the static margin, that is equal to $2.4 \%$.

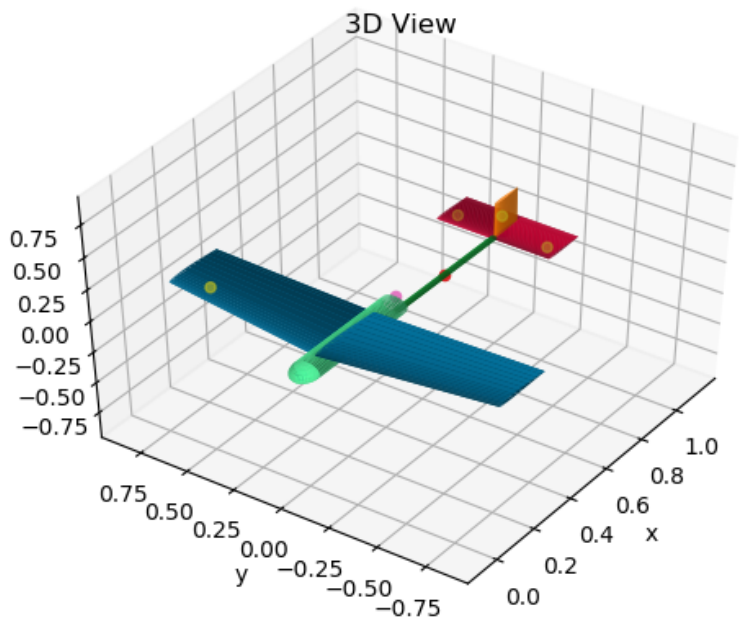

Figure 6: Aircraft configuration at iteration number 1.

An intermediate condition can be seen in Fig. 7 obtained at step 2000. It is a configuration already very similar to the final concept, shown in Fig. 8. The sweep angle is already similar, and the tail boom is shorter than the initial one. The final span is $1.289 \mathrm{~m}$, with a small taper, with chord varying from 0.150 
$\mathrm{m}$ at the root to $0.116 \mathrm{~m}$ at the tip. The mass practically did not change after iteration 1400 , reaching a value near $1.71 \mathrm{~kg}$. The final convergence was achieved at iteration 4150 because no better value was obtained after 1000 steps.

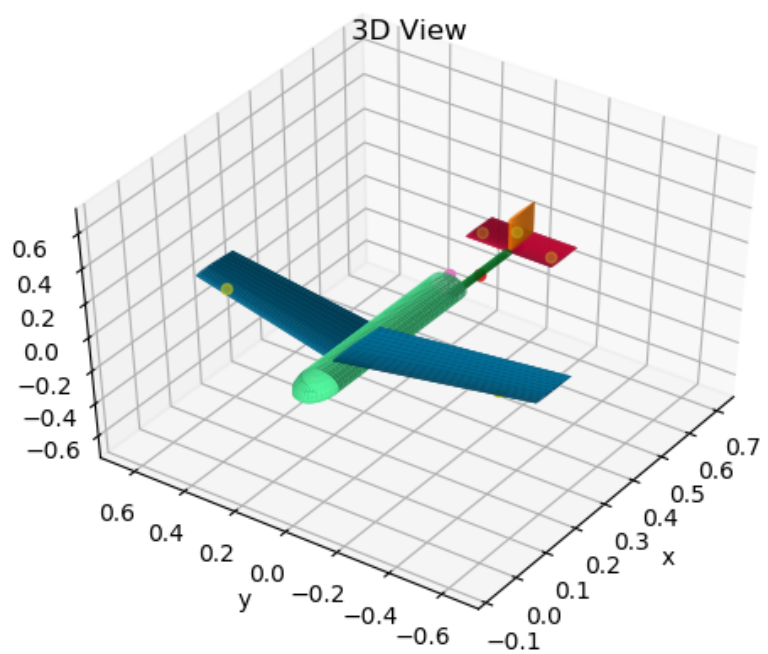

Figure 7: Aircraft configuration at iteration number 2000.

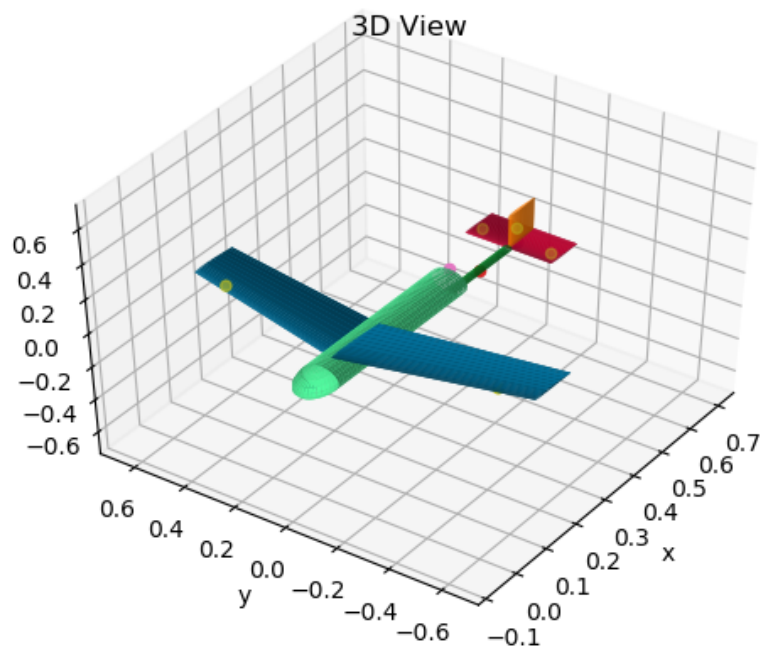

Figure 8: Aircraft final configuration.

The optimized design variables vector is presented in Table 8 The star * indicates that the specific design variable stopped into the lower bound defined in Table 6 . In this case, the fuselage fineness ratio gets lower because the volume is constant, and thus the MDO process tries to control the CG position and the inertia values, due to the static margin constraint.

Some of the output data from the optimization problem are presented in Table 9 In the aerodynamics block, $i_{w}$ and $i_{H T}$ are the incidence angles of the wing and horizontal tail, respectively, $C_{D_{0}}$ is induced drag coefficient and $k$ is defined as $1 /(\pi e A R)$, where $e$ is Oswald's efficiency. In the performance block, $E$ is endurance and $R$ is range estimate. In the stability block, $C_{l_{\beta}}$ is the rolling moment due to sideslip derivative and $C_{y_{\beta}}$ is the side force due to sideslip derivative.
Table 8: optimized design variables obtained in the case study.

\begin{tabular}{llll}
\hline № & Variable & $x_{\text {gbest }}$ & Unit \\
\hline 1 & $A R$ & 9.67 & {$[-]$} \\
2 & $S$ & 0.17 & {$\left[\mathrm{~m}^{2}\right]$} \\
3 & $\lambda$ & 0.77 & {$[-]$} \\
4 & $\Lambda_{L E}$ & 8.47 & {$[\mathrm{deg}]$} \\
5 & $x_{r_{w}} / l_{f u s}$ & 0.13 & {$[-]$} \\
6 & $\lambda_{f u s}$ & $3^{*}$ & {$[-]$} \\
7 & $A R_{H T}$ & 3.69 & {$[-]$} \\
8 & $A R_{V T}$ & 2.17 & {$[-]$} \\
9 & $l_{H T} / \bar{c}$ & 3.87 & {$[-]$} \\
\hline
\end{tabular}

Table 9: Output data from the optimization problem in study.

\begin{tabular}{llll}
\hline Area/No & Parameter & Value & Unit \\
\hline \multicolumn{4}{c}{ Mass properties } \\
1 & $\begin{array}{l}M_{\text {tot }} \\
\text { Aerodynamics }\end{array}$ & 1.71 & {$[\mathrm{~kg}]$} \\
11 & $i_{w}$ & 3.23 & {$[\mathrm{deg}]$} \\
11 & $i_{H T}$ & 1.31 & {$[\mathrm{deg}]$} \\
11 & $C_{D_{0}}$ & 0.055 & {$[-]$} \\
12 & $k$ & 0.041 & {$[-]$} \\
& Performance & & \\
34 & $E$ & 36 & {$[\mathrm{~min}]$} \\
34 & $R$ & 44 & {$[\mathrm{~km}]$} \\
& Stability & & \\
34 & $C_{m_{\alpha}}$ & -0.584 & {$[1 / \mathrm{rad}]$} \\
34 & $C_{n_{\beta}}$ & 0.0 & {$[1 / \mathrm{rad}]$} \\
34 & $C_{l_{\beta}}$ & -0.079 & {$[1 / \mathrm{rad}]$} \\
34 & $C_{y_{\beta}}$ & -0.412 & {$[1 / \mathrm{rad}]$} \\
34 & $S M$ & 12.5 & {$[\%]$} \\
\hline
\end{tabular}

The solution respected all imposed constraints. The output data from aerodynamics are in the expected order of magnitude. The moment coefficient derivative in respect to the angle of attack $C_{m_{\alpha}}$ is negative, ensuring longitudinal static stability to the aircraft. The yaw moment coefficient derivative with respect to the sideslip angle $C_{n_{\beta}}$ is zero. It respected the imposed constraint, but it was different from the expected $\left(C_{n_{\beta}}>0\right)$ to ensure directional static stability.

The eigenvalues of the longitudinal and lateral directional dynamics, describing short period characteristics for example, are presented below:

$$
\begin{gathered}
\lambda_{\text {long }}=\left\{\begin{array}{c}
-6.8 \pm 7.32 j \\
-9.21 \times 10^{-2} \pm 0.551 j \\
-1.31 \times 10^{-4}+0 . j
\end{array}\right\} \\
\lambda_{\text {lat }}=\left\{\begin{array}{c}
-116.43+0 . j \\
-0.247 \pm 1.6 j \\
-3.17+0 . j
\end{array}\right\}
\end{gathered}
$$




\section{Concluding remarks}

This paper describes the development of a tool for remotely piloted aircraft conceptual design considering a MDO framework integrated with a PSO optimization algorithm. The tool embraces diverse aeronautical engineering disciplines (aerodynamics, stability, flight dynamics, etc.), all coupled into a single driver code that handles all design information. This way, it's easier to perceive how an integrated process can facilitate decisions among a multidisciplinary team.

A numerical study is presented, aiming to obtain a concept of a small RPA. The case study considers the definition of multiple variable constraints, which requires certain expertise by the designer. Sometimes, the range of each variable differ considerably from the usual general aviation values, usually proposed by consecrated references, like Roskam [21] and Raymer [2]. The number of subsystems, and consequently, parts, decrease when the designer's team is dealing with unmanned aircraft. This happens, mainly, because it's not necessary to add life support and comfort systems. Also, dimensions are smaller, and the aerodynamics parameters are affected, for example. Therefore, to consider the constraints of RPA missions using known general aviation methodologies is still a challenging task.

This is an ongoing development work. The disciplines analyses are implemented in Python language in form of independent packages, called by the main code. So, new disciplines can be easily added or the ones already present can be improved. The same approach is applicable to the optimization method, once the RPA design code is also a module to be called in the routine.

\section{List of Abbreviations and Acronyms \\ CAD Computer Aided Design. \\ MAV micro aerial vehicle. \\ MDO Multidisciplinary Design optimization. \\ RPA remotely piloted aircraft. \\ RPAS remotely piloted aircraft system. \\ UAV unmanned aerial vehicle.}

\section{References}

[1] W. J. Vankan, W. F. Lammen, and R. Maas. Metamodeling and multi-objective optimization in aircraft design. Technical report, National Aerospace Laboratory, 2010.

[2] Daniel P. Raymer. Aircraft Design: A Conceptual Approach. AIAA education series. American Institute of Aeronautics and Astronautics (AIAA), Washington, D.C., 4th edition, 2006. ISBN 1563478293.

[3] Peter De Baets and Dimitri Mavris. Potential formulation for aeroelastic constraint analysis in a conceptual design environment. In 43rd AIAA/ASME/ASCE/AHS/ASC Structures, Structural Dynamics, and Materials Conference, Structures, Structural Dynamics, and Materials and Co-located Conferences. American Institute of Aeronautics and Astronautics, April 2002. doi: 10.2514/6.2002-1295.
[4] Plamen Roglev. MDO Framework for Conceptual Design of Closed Wing UAV. International Journal of Scientific Research Engineering \& Technology (IJSRET), 2(8):526-531, 2013.

[5] Joaquim R. R. A. Martins and Andrew B. Lambe. Multidisciplinary Design Optimization: A Survey of Architectures. AIAA Journal, 51(9):2049-2075, 2013. ISSN 0001-1452. doi: 10.2514/1.J051895.

[6] B. Leonard. Department of Defense Dictionary of Military and Associated Terms: As Amended Through April 2010. DIANE Publishing Company, 2011. ISBN 9781437938203.

[7] Reg Austin. Unmanned Aircraft Systems: UAVS Design, Development and Deployment. John Wiley \& Sons, Inc., Chippenham, Wiltshire, 2010.

[8] Jaroslaw Sobieszczanski-Sobieski and Raphael T. Haftka. Multidisciplinary aerospace design optimization - survey of recent developments. In AIAA 34th Aerospace Sciences Meeting and Exhibit, Reno, NV, Jan 15-18 1996.

[9] Andras Sobester and Andy Keane. Multidisciplinary design optimization of uav airframes. In 47th AIAA/ASME/ASCE/AHS/ASC Structures, Structural Dynamics, and Materials Conference, Structures, Structural Dynamics, and Materials and Co-located Conferences. American Institute of Aeronautics and Astronautics, May 2006. doi: 10.2514/6.2006-1612.

[10] Giuseppe Landolfo. Aerodynamic and structural design of a small nonplanar wing uav. Master's thesis, University of Dayton, 2008.

[11] David Lundstrom, Kristian Amadori, and Petter Krus. Distributed framework for micro aerial vehicle design automation. In 46th AIAA Aerospace Sciences Meeting and Exhibit, Aerospace Sciences Meetings. American Institute of Aeronautics and Astronautics, January 2008. doi: $10.2514 / 6.2008-140$.

[12] Jeremy Agte, Olivier de Weck, Jarowlaw Sobieszczanski-Sobieski, Paul Arendsen, Alan Morris, and Martin Spieck. MDO: assesment and direction for advancement - an opinion of one international group. Structural and Multidisciplinary Optimization, 40: 17-33, 2010.

[13] Fabricio Ardais Medeiros. Desenvolvimento de um veículo aéreo não tripulado para aplicação em agricultura de precisão. Master's thesis, Universidade Federal de Santa Maria, 2007.

[14] R Elmendorp, R Vos, and Gianfranco La Rocca. A conceptual design and analysis method for conventional and unconventional airplanes. In 29th Congress of the International Council of the Aeronautical Sciences, ICAS. International Council of Aeronautical Sciences, 2014. 
[15] Dean E. Bryson, Christopher R. Marks, Ryan M. Miller, and Markus P. Rumpfkeil. Multidisciplinary design optimization of quiet, hybrid-electric small unmanned aerial systems. Journal of Aircraft, 53(6):1959-1963, June 2016. ISSN 0021-8669. doi: 10.2514/1.C033455.

[16] Majid Hosseini, Mehran Nosratollahi, and Hossein Sadati. Multidisciplinary design optimization of unmanned aerial vehicle under uncertainty. Journal of Aerospace Technology and Management, 2017. doi: http://dx.doi. org/10.5028/jatm.v9i2.725.

[17] Athanasios Papageorgiou, Johan Ölvander, and Kristian Amadori. Development of a multidisciplinary design optimization framework applied on uav design by considering models for mission, surveillance, and stealth performance. In 18th AIAA/ISSMO Multidisciplinary Analysis and Optimization Conference, AIAA AVIATION Forum. American Institute of Aeronautics and Astronautics, June 2017. doi: 10.2514/6.2017-4151.

[18] M.H. Sadraey. Aircraft Design: A Systems Engineering Approach. Aerospace Series. Wiley, 2012. ISBN 9781119953401 .

[19] Jay Gundlach. Designing Unmanned Aircraft Systems: A Comprehensive Approach. American Institute of Aeronautics and Astronautics, Inc., Reston, Virginia, 2012.

[20] Snorri Gudmundsson. General Aviation Aircraft Design: Applied Methods and Procedures. Elsevier, Waltham, Massachusetts, 1st edition, 2014.

[21] Jan Roskam. Airplane Design. Roskam Aviation and Engineering Corporation, 1985.

[22] Lance W. Traub. Range and endurance estimates for battery-powered aircraft. Journal of Aircraft, 48(2): 703-707, 2011. doi: 10.2514/1.C031027.

[23] Jan Roskam. Methods for Estimating Stability and Control Derivatives of Conventional Subsonic Airplanes. Roskam aviation and engineering corporation, 1983.

[24] M.V. Cook. Flight Dynamics Principles: A Linear Systems Approach to Aircraft Stability and Control. Elsevier aerospace engineering series. Elsevier Science, 2011. ISBN 9780080550367.

[25] Xin-She Yang. Engineering Optimization: An Introduction with Metaheuristic Applications. John Wiley \& Sons, Inc., Hoboken, New Jersey, 2010.

[26] UAS Europe. Spy owl 200, 2019. URL http://www . uas-europe.se/index .php/ products-mobile/spy-owl-200-mobile. Online; Acessed: 10 set. 2018.

[27] Leland M. Nicolai and Grant E. Carichner. Fundamentals of Aircraft and Airship Design, volume Vol. I - Aircraft Design. American Institute of Aeronautics and Astronautics, Inc., 2010. 